\title{
Comparing Two Waist-to-Height Ratio Measurements with Cardiometabolic Risk Factors among Youth with Diabetes
}

Lenna L. Liu ${ }^{1, *}$, Henry S. Kahn ${ }^{2}$, David J. Pettitt ${ }^{3}$, Nora F. Fino ${ }^{4}$, Tim Morgan ${ }^{4}$, David M. Maahs ${ }^{5}$, Nancy A. Crimmins ${ }^{6}$, Archana P. Lamichhane ${ }^{7}$, Angela D. Liese ${ }^{8}$, Ralph B. D’Agostino Jr. ${ }^{4}$ and Ronny A. Bell ${ }^{4}$

${ }^{1}$ University of Washington and Seattle Children's Hospital, Seattle, WA 98122, USA

${ }^{2}$ Centers for Disease Control and Prevention, Mail Stop K-10, 4770 Buford Highway, NE Atlanta, GA 30341 3717, USA

${ }^{3} 1001$ Arbolado Road, Santa Barbara, CA 93103, USA

${ }^{4}$ Division of Public Health Sciences, Wake Forest School of Medicine, Medical Center Boulevard, WinstonSalem, NC 27157, USA

${ }^{5}$ Barbara Davis Center for Childhood Diabetes, University of Colorado Denver, P. O. Box 6511, Mail Stop A140, Aurora, CO 80045, USA

${ }^{6}$ Cincinnati Children's Hospital Medical Center, 3333 Burnet Avenue, Mail Location 7012, Cincinnati, OH 45229, USA

${ }^{7}$ Department of Nutrition, University of North Carolina at Chapel Hill, 2211 McGavran Greenberg, CB 7461, Chapel Hill, NC 27599-7461, USA

${ }^{8}$ Department of Epidemiology and Biostatistics and Center for Research in Nutrition and Health Disparities, Arnold School of Public Health, University of South Carolina, 800 Sumter Street, Columbia, SC 29208, USA

Abstract: Background: Waist circumference (WC) is commonly measured by either the World Health Organization (WHO) or National Health and Nutrition Examination Survey (NHANES) protocol.

Objective: Compare the associations of WHO vs. NHANES WC-to-height ratio (WHtR) protocols with cardiometabolic risk factors (CMRFs) in a sample of youth with diabetes.

Methods: For youth (10-19 years old with type 1 [N=3082] or type 2 [N=533] diabetes) in the SEARCH for Diabetes in Youth Study, measurements were obtained of WC (by two protocols), weight, height, fasting lipids (total cholesterol, triglycerides, HDL cholesterol, Non-HDL cholesterol) and blood pressures. Associations of CMRFs with WHO and NHANES WHtR were modeled stratified by body mass index (BMI) percentiles for age/sex: lower BMI $\left(<85^{\text {th }} \mathrm{BMI}^{\mathrm{B}}\right.$ percentile; $\mathrm{N}=2071) v s$. higher $\mathrm{BMI}\left(\geq 85^{\text {th }}\right.$ percentile; $\left.\mathrm{N}=1594\right)$.

Results: Among lower-BMI participants, both NHANES and WHO WHtR were associated $(p<0.005)$ with all CMRFs except blood pressure. Among higher-BMI participants, both NHANES and WHO WHtR were associated $(p<0.05)$ with all CMRFs. WHO WHtR was more strongly associated $(p<0.05)$ than NHANES WHtR with triglycerides, non-HDL cholesterol, and systolic blood pressure in lower-BMI participants. Among high-BMI participants, WHO WHtR was more strongly associated $(p<0.05)$ than NHANES WHtR with triglycerides and systolic blood pressure.

Conclusion: Among youth with diabetes, WHtR calculated from either WC protocol captures cardiometabolic risk. The WHO WC protocol may be preferable to NHANES WC.

Keywords: Waist Circumference, Body Mass Index, Diabetes, Cardiovascular Risk.

\section{BACKGROUND}

The association between central adiposity and cardiometabolic risk factors (CMRFs) among adults with diabetes is well known; however, less is known about this association among youth.

While body mass index (BMI), including transformations such as BMI percentiles or z-scores,

*Address correspondence to this author at the Odessa Brown Children's Clinic, 2101 E Yesler Way, Seattle, WA 98122, USA; Tel: 206-987-7218;

Fax: 206-329-9764; E-mail: lennall@uw.edu has been used as a clinical marker for cardiometabolic risk, inherent limitations are recognized, particularly the lack of differentiation in body fat distribution. Given the concern about total abdominal or visceral (intraabdominal) fat, the waist-to-height ratio ( $\mathrm{WHtR})$ has been proposed as an additional or alternative risk indicator [1], with the simple public health message to "Keep your waist circumference (WC) to less than half your height" [2]. In growing children, WHtR makes more inherent sense than WC alone given their ongoing height growth. More recently, Kahn et al., using data in a sample of youth aged 11-12 years from 
the HEALTHY Study, showed that assessments by WHtR can provide cardiometabolic risk estimates similar to conventional BMI z-scores without requiring reference to a normative growth chart [3].

WC measures differ based on anthropometric protocols recommended by the World Health Organization (WHO) and by the National Health and Nutrition Examination Survey (NHANES). However, neither has been adopted clinically as a consistent standard measure. In a previous comparison of $\mathrm{WHO}$ and NHANES WC measures among participants of the SEARCH for Diabetes in Youth study ( $\mathrm{N}=6248$; aged 225 years), the mean NHANES WC was found to be greater than the mean WHO WC $(76.3 \mathrm{~cm} v s .71 .9 \mathrm{~cm})$ [4]. These differences were greater for females, among older participants, and in those with higher BMI. In both sexes and across ages, the WCs from both methods were highly correlated with BMI z-scores and no evidence was found that one of these two methods was a better indicator of BMI z-score. However, that study did not examine the association of WC or WHtR to CMRFs.

There has previously been some suggestion that WHO WC is a better predictor of CMRFs than NHANES WC. Johnson et al. [5] compared four waist measures to CMRFs in a population of 8-17 year old Canadian youth with BMIs $\geq 85^{\text {th }}$ percentile referred for weight management. They found that waist measurements at the narrowest waist or midpoint between the floating rib and iliac crest were more closely associated with metabolic risk. Ma et al. [6] also recently examined the difference between WC-mid (analogous to WHO) and WC-iliac crest (IC, analogous to NHANES) in predicting CMRFs and diabetes risk among Asian adults and found that WC-mid was more closely related to visceral fat area (by CT) and CMRFs.

The SEARCH for Diabetes in Youth Study provides a unique opportunity to compare various measures of adiposity in a large sample of racially and ethnically diverse participants with types 1 (T1D) or 2 (T2D) diabetes. Using $\mathrm{WHtR}$ as our primary adiposity measure, we examined the association between WHtR and CMRFs and compared WHO and NHANES protocols to test if WHO WC was more closely associated with CMRFs than NHANES WC.

\section{METHODS}

\section{Study Design and Population}

SEARCH is a multicenter population-based study that started in 2001 and ascertains youth aged $<20$ years with clinically diagnosed diabetes, excluding those with gestational diabetes [7]. Cases were ascertained from geographically defined populations in Ohio, Colorado, South Carolina and Washington; from Indian Health Service beneficiaries from four American Indian populations; and from enrollees in a managed health care plan in California. Institutional review board(s) for each site approved the study protocol. Youth whose diabetes was not secondary to other conditions were invited to a SEARCH study visit. Females who said they were possibly pregnant were excluded from these analyses.

\section{Waist Measurement}

Youth aged 10-19 years with physician-diagnosed diabetes that was prevalent in 2001 or incident between 2002 and 2008, excluding 2007, were invited to complete an in-person visit that included collecting a blood sample and anthropometric assessments conducted by centrally trained research staff (registered cases in SEARCH Phase 2 from 2006-2010 were only invited for visits in alternate years). WC was systematically measured by two protocols during each examination. Participants missing one of the WC protocol measurements were excluded $(n=120)$. For each measurement, the measuring tape was positioned parallel to the floor with the participant standing, abdomen relaxed, arms at the sides, feet together and facing the observer with the waist exposed.

In the method used for NHANES, WC was assessed just above the right iliac crest at the midaxillary line [8]. In the method recommended by WHO, WC was taken with the tape midway between the lowest rib margin and the iliac crest at the mid-axillary line [9]. Measurement by the NHANES method was always done first and measurement by the WHO method was done last, and were taken by study personnel trained and certified centrally on both methods. For each method, two measurements were taken and, if they differed by more than $1.0 \mathrm{~cm}$, a third measurement was performed. A third measure was required in $130(3.6 \%)$ of $\mathrm{WHO}$ waist measures and $164(4.5 \%)$ of NHANES waist measures. Data from the mean of the two or three measurements for each method were used for analyses.

Height was measured using a stadiometer. Two height measures were taken for each participant and the average of the measures was calculated. WHtR was calculated by dividing the average of the waist measures (in centimeters) by the average of the height measures (in centimeters). 


\section{Cardiometabolic Risk Factors}

At the in-person visit, blood was drawn after fasting for at least eight hours to measure lipids (total cholesterol [TC], low-density lipoprotein cholesterol [LDL-C], high-density lipoprotein cholesterol [HDL-C], and triglycerides [TG]). We also determined non-HDLC by subtracting HDL-C from TC. Laboratory samples were obtained only if there had been no episode of diabetic ketoacidosis within the prior month. Specimens were processed locally at the sites and then shipped within 24 hours to the central laboratory (Northwest Lipid Metabolism and Diabetes Research Laboratories, University of Washington, Seattle, WA), where they were analyzed. Measurements of TC, HDL-C, and triglycerides were performed enzymatically on a Hitachi 917 autoanalyzer (Boehringer Mannheim Diagnostics, Indianapolis, Indiana). LDL-C levels were calculated by the Friedewald equation for individuals with triglyceride levels $<400 \mathrm{mg} / \mathrm{dL}$ [10] and by Lipid Research Clinics Beta Quantification [11] for those with triglyceride levels $\geq 400 \mathrm{mg} / \mathrm{dL}$.

Three blood pressure measurements were obtained during the in-person visit using a portable mercury manometer [12]. The average of the three measures for systolic and diastolic blood pressure was used for analysis.

\section{Statistical Analysis}

To characterize our analytic sample, we calculated descriptive statistics by diabetes type (T1D or T2D) and BMI categories (lower BMI: BMI percentile $<85 \%$ or higher BMI: BMI percentile $\geq 85 \%$ ) for age and sex [13]. Means and standard deviations were used for continuous variables, while frequencies and percentages were used for categorical variables. To evaluate the agreement between the NHANES WHtR and the WHO WHtR, we dichotomized each WHtR indicator as values $\leq 0.5$ and $>0.5$ and calculated a Cohen's Kappa statistic. This stratification is consistent with the analyses by McCarthy and Ashwell [2] and is generally consistent with the WHtR boundary value for adults.

The primary outcome variables were CMRFs: triglycerides, log-transformed (Log-TG); total, HDL-, and LDL-cholesterol (TC, HDL-C, LDL-C); systolic and diastolic blood pressure (SBP, DBP). To describe any differences in these factors by NHANES and WHO WHtR, we compared the mean CMRFs by the dichotomous $\mathrm{WHtR}$ variables using two sample t-tests.
Lastly, linear regression was used to determine the partial correlation between the CMRF outcomes and each continuous WHtR measure, after adjusting for continuous age, diabetes type, sex, and race. A squared term for age was also included to account for nonlinearity in CMRF outcomes as participants increased in age. To determine if the NHANES WHtR and WHO WHtR were equally correlated with the CMRF outcomes, we tested for differences [14] in the partial correlations of each outcome and the NHANES WHtR and WHO WHtR measures. All statistical analyses were performed in SAS 9.4 (Cary, NC), and a $p$-value of $<0.05$ was considered significant.

\section{RESULTS}

Participant characteristics for the 3615 youth with diabetes are shown in Table 1, with stratifications by BMI percentile categories (lower BMl: $n=2071$ vs. higher BMI: $n=1544$ ) and sex. When analyses were stratified by DM type, not only was the number of youth with T2D much smaller than the number with T1D, but they almost all had $\mathrm{BMI}>95^{\text {th }}$ percentile (over $80 \%$, data not shown). Therefore, we present our results stratified by BMI percentile categories instead of DM type. NHANES WC and WHtR were consistently larger than respective WHO measures. Youth in the lower BMI category had WHtRs generally $\leq 0.5(90.2 \%$ of youth with T1D had NHANES WHtR $\leq 0.5$ and $98.7 \%$ for $\mathrm{WHO}$ WHtR) and youth with higher BMI had WHtRs $>0.5(98.4 \%$ of youth with T2D had NHANES WHtR $\geq 0.5$ and $93.6 \%$ for $\mathrm{WHO}$ WHtR).

The concordance between WHtR $(\leq 0.5,>0.5)$ using the NHANES and WHO WC protocols is shown in Table 2. Given the skewed distribution of BMI percentiles towards overweight and obesity among youth with larger BMls, only $23 \%$ of youth $(20.1 \%$ for males, $25.3 \%$ for females) had discordance where the NHANES WHtR was $>0.5$ and the $\mathrm{WHO} W H t R$ was $\leq 0.5$ (Kappa=0.55 in males, Kappa=0.28 in females); only $0.4 \%$ had discordance in the other direction (NHANES WHtR $\leq 0.5$ and WHO WHtR>0.5). For youth with BMIs below than the $85^{\text {th }}$ percentile, approximately $3 \%$ of males and $15 \%$ of females had discordance where the NHANES WHtR was $>0.5$ and the WHO WHtR was $\leq 0.5$ (Kappa $=0.27$ and 0.18 , respectively). Although the discordance in the other direction (NHANES $\mathrm{WHtR} \leq 0.5$ and $\mathrm{WHO} \mathrm{WHtR}>0.5$ ) was very small (less than $0.1 \%$ ) among youth with T1D, it was not zero. Overall, the concordance between WHtR using NHANES and WHO was better for males than females. 
Table 1: Selected Patient Characteristics by BMI Percentile Category ( $<85 \%, \geq 85 \%)$ and Gender

\begin{tabular}{|c|c|c|c|c|}
\hline & \multicolumn{2}{|c|}{ BMI Percentile < 85\% } & \multicolumn{2}{|c|}{ BMI Percentile $\geq 85 \%$} \\
\hline $\mathrm{N}(\%)$ & 993 (47.9) & $1078(52.1)$ & $833(54.0)$ & $711(46.0)$ \\
\hline \multicolumn{5}{|l|}{ Age Group (Years, \%) } \\
\hline $10-12$ & $457(46.0)$ & $386(35.8)$ & $260(31.2)$ & $240(33.8)$ \\
\hline \multicolumn{5}{|l|}{ Race/Ethnicity (\%)* } \\
\hline NHW & $760(76.5)$ & $872(80.9)$ & $412(49.5)$ & $430(60.5)$ \\
\hline Hispanic & $108(10.9)$ & $109(10.1)$ & $150(18.0)$ & $135(19.0)$ \\
\hline$A A$ & $87(8.8)$ & $71(6.6)$ & $222(26.7)$ & $105(14.6)$ \\
\hline $\begin{array}{l}\text { NHANES WC } \\
\text { (Mean, SD) }\end{array}$ & $72.5(8.0)$ & $72.4(7.4)$ & $97.3(18.2)$ & $95.5(19.1)$ \\
\hline $\begin{array}{l}\text { WHO WC } \\
\text { (Mean, SD) }\end{array}$ & $66.6(6.4)$ & $69.5(6.7)$ & $88.9(15.4)$ & $89.6(17.3)$ \\
\hline NHANES WHtR (Mean, SD) & $0.463(0.039)$ & $0.442(0.030)$ & $0.604(0.105)$ & $0.572(0.096)$ \\
\hline $\begin{array}{l}\text { WHO WHtR } \\
\text { (Mean, SD) }\end{array}$ & $0.426(0.032)$ & $0.424(0.028)$ & $0.553(0.091)$ & $0.537(0.088)$ \\
\hline \multicolumn{5}{|l|}{ Diabetes Type (\%) } \\
\hline Type 1 & $971(97.8)$ & $1065(98.8)$ & $531(63.8)$ & $515(72.4)$ \\
\hline
\end{tabular}

${ }^{*}$ Race/ethnicity category abbreviations: NHW: Non-Hispanic White; AA: African American; API: Asian Pacific Islander; Al/AN: American Indian/Alaska Native.

Table 2: Agreement of WHtR Categories Measured Using NHANES and WHO Protocols by Gender

\begin{tabular}{|c|c|c|c|c|c|}
\hline BMI Percentile <85\% & & \multicolumn{2}{|c|}{ Male } & \multicolumn{2}{|c|}{ Female } \\
\hline & & \multicolumn{2}{|c|}{ WHO } & \multicolumn{2}{|c|}{ WHO } \\
\hline \multirow{4}{*}{ NHANES } & & $\begin{array}{l}\leq 0.5 \\
\mathrm{n}(\%)\end{array}$ & $\begin{array}{l}>0.5 \\
\mathrm{n}(\%)\end{array}$ & $\begin{array}{l}\leq 0.5 \\
\mathrm{n}(\%)\end{array}$ & $\begin{array}{l}>0.5 \\
\mathrm{n}(\%)\end{array}$ \\
\hline & $\leq 0.5$ & $1034(95.9)$ & $0(0)$ & $821(82.7)$ & $1(0.10)$ \\
\hline & $>0.5$ & $37(3.43)$ & $7(0.7)$ & $151(15.2)$ & $20(2.0)$ \\
\hline & Kappa $(95 \%$ CI) & \multicolumn{2}{|c|}{$\begin{array}{c}0.27 \\
(0.11,0.42)\end{array}$} & \multicolumn{2}{|c|}{$\begin{array}{c}0.18 \\
(0.11,0.25)\end{array}$} \\
\hline BMI Percentile $\geq 85 \%$ & & \multicolumn{2}{|c|}{ WHO } & \multicolumn{2}{|c|}{ WHO } \\
\hline \multirow{4}{*}{ NHANES } & & $\begin{array}{l}\leq 0.5 \\
\mathrm{n}(\%)\end{array}$ & $\begin{array}{l}>0.5 \\
\mathrm{n}(\%)\end{array}$ & $\begin{array}{l}\leq 0.5 \\
\mathrm{n}(\%)\end{array}$ & $\begin{array}{l}>0.5 \\
\mathrm{n}(\%)\end{array}$ \\
\hline & $\leq 0.5$ & $150(21.1)$ & $2(0.3)$ & $64(7.7)$ & $4(0.5)$ \\
\hline & $>0.5$ & $143(20.1)$ & $416(58.5)$ & $211(25.3)$ & 554 (66.5) \\
\hline & Kappa $(95 \%$ Cl) & \multicolumn{2}{|c|}{$\begin{array}{c}0.55 \\
(0.49,0.61)\end{array}$} & \multicolumn{2}{|c|}{$\begin{array}{c}0.28 \\
(0.22,0.34)\end{array}$} \\
\hline
\end{tabular}

The associations between CMRFs and WHtR according to the NHANES and WHO protocol, stratified by BMI percentile categories, are shown in Table 3 . When analyzed separately by sex, results did not differ; 
Table 3: Associations between Cardiometabolic Risk Factors and WHtR by BMI Percentile Category

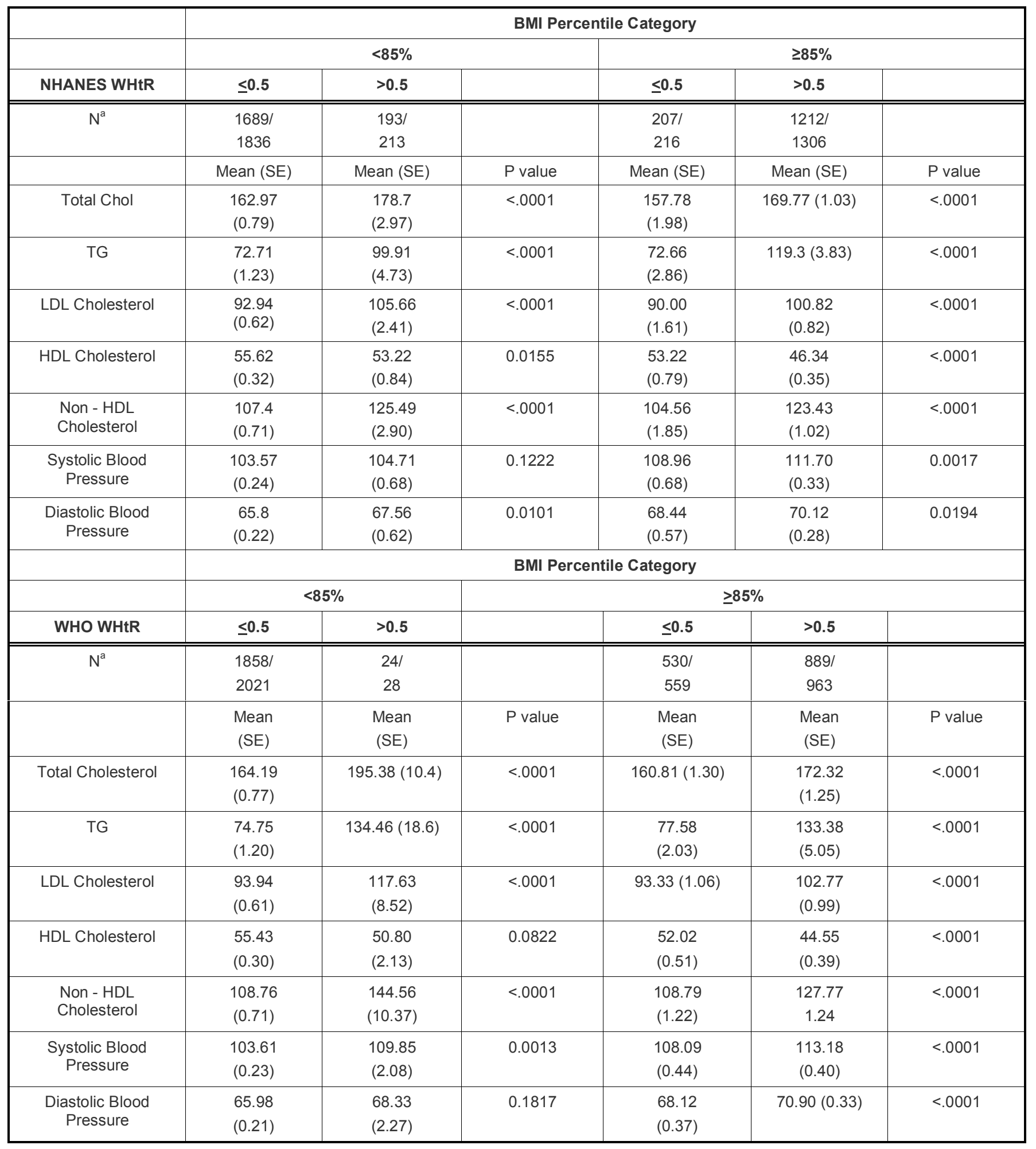

${ }^{a}$ Sample size for lipid measures/Sample size for blood pressure measures.

therefore, results are presented for the two sexes combined. Youth with WHtRs $>0.5$ (both NHANES and WHO) had significantly higher TC, TG, LDL-C, and non-HDL-C than youth with WHtRs $\leq 0.5$ across BMI percentile categories.
Finally, linear regression results of the association between NHANES and WHO WHtR and individual CMRFs, adjusted for age, diabetes type, race and ethnicity, and sex, and stratified by BMI percentiles, are shown in Table 4. When analyzed separately, results 
Table 4: Association* between Two Measures of WHtR and Cardiometabolic Risk Factors, Stratified by BMI Percentile Category

\begin{tabular}{|c|c|c|c|c|c|c|}
\hline & \multicolumn{2}{|c|}{ BMI Percentile <85\% } & \multirow[t]{2}{*}{ P Value $^{\mathrm{a}}$} & \multicolumn{2}{|c|}{ BMI Percentile $\geq 85 \%$} & \multirow[t]{2}{*}{ P Value } \\
\hline & NHANES WHtR & $\begin{array}{l}\text { WHO } \\
\text { WHtR }\end{array}$ & & NHANES WHtR & $\begin{array}{l}\text { WHO } \\
\text { WHtR }\end{array}$ & \\
\hline Total Cholesterol & $0.12(<0.001)$ & $0.13(<0.001)$ & 0.24 & $0.05(0.04)$ & $0.07(0.008)$ & 0.15 \\
\hline Log -Triglycerides & $0.13(<0.001)$ & $0.18(<0.001)$ & $<0.001$ & $0.14(<0.001)$ & $0.17(<0.001)$ & 0.007 \\
\hline LDL-Cholesterol & $0.13(<0.001)$ & $0.14(<0.001)$ & 0.21 & $0.07(0.01)$ & $0.08(0.002)$ & 0.23 \\
\hline HDL-Cholesterol & $0.07(0.003)$ & $0.09(0.0001)$ & 0.14 & $0.18(<0.001)$ & $0.19(<0.001)$ & 0.17 \\
\hline $\begin{array}{l}\text { Non - HDL } \\
\text { Cholesterol }\end{array}$ & $0.16(<0.001)$ & $0.18(<0.001)$ & 0.048 & $0.11(<0.001)$ & $0.13(<0.001)$ & 0.07 \\
\hline $\begin{array}{l}\text { Systolic Blood } \\
\text { Pressure }\end{array}$ & $0.02(0.40)$ & $0.06(0.006)$ & 0.4 & $0.09(0.0003)$ & $0.12(<0.001)$ & 0.02 \\
\hline $\begin{array}{l}\text { Diastolic Blood } \\
\text { Pressure }\end{array}$ & $0.04(0.04)$ & $0.04(0.11)$ & 0.31 & $0.07(0.01)$ & $0.09(0.001)$ & 0.17 \\
\hline
\end{tabular}

${ }^{*}$ Partial correlation ( $p$ value for association with WHtR); adjusted for diabetes type, age, age squared, race/ethnicity, and gender.

${ }^{a} \mathrm{P}$-value for test that the partial correlation with NHANES WHtR is the same as the partial correlation with WHO WHtR.

did not differ by sex; therefore, the results were combined and sex-adjusted in the model. For both BMI percentile categories, both WHtR measures were associated with most of the CMRFs. In youth in the lower BMI category, compared to the NHANES WHtR, the WHO WHtR was more strongly associated with logTG and non-HDL-C $(p<0.001$ and 0.048$)$. For LDL-C, HDL-C, and DBP, however, the stronger association of the WHO WHtR was not statistically significant ( $p$ range 0.14 to 0.4 ). Among youth in the higher BMI category, partial correlation coefficients were significant for both NHANES and WHO WHtR for all CMRFs NHANES WHtR. The WHO WHtR was more strongly associated with log-TG and SBP.

\section{DISCUSSION}

As seen in previous studies, NHANES and WHO WC measures differ significantly; NHANES methodology leads to larger WC measurements than those of WHO. This is consistent with findings in most studies in adults and those including children and adolescents [6, 15-17]. For example, Patry-Parisien et al. recently compared WHO and NHANES WC measurements among Canadian aged 3-79 years and found significant differences between the two measures, again with NHANES measures being greater than those from the WHO protocol [16].

We have previously reported rates of overweight and obesity from earlier analyses of data from the SEARCH population [18]. Among youth with T2D, the prevalence of overweight was $10.4 \%$ and of obesity was $79.4 \%$; among youth with T1D, $22.1 \%$ were overweight and $12.6 \%$ were obese. We found similar ranges in this SEARCH cohort. Using WHtR as our measure of adiposity, the distribution of WHtR differed between youth with T1D and T2D as expected given these BMI percentile distributions. Notably among youth with T1D, however, there were youth with both low $(\mathrm{WHtR} \leq 0.5)$ and high $(\mathrm{WHtR}>0.5) \mathrm{WHtRs}$ and those with high WHtRs had higher CMRFs.

WHtR was associated with CMRFs among both lower and higher BMl categories. The WHO WHtR measurement was more strongly associated with several of the CMRFs (triglycerides, non-HDL cholesterol, and systolic blood pressure) relative to the NHANES WHtR. While our study cannot demonstrate why the WHO measure is more strongly associated to CMRFs compared to NHANES, we suggest some explanations based on the distinct anatomic differences in WC measurement methodology. As described in our methods, NHANES WC is assessed just above the right iliac crest at the mid-axillary line [8] in contrast to WHO WC which is measured midway between the lowest rib margin and the iliac crest at the mid-axillary line [9]. It is possible that the NHANES WC measurement is limited or constrained by the fixed bony anatomy of the pelvis whereas the WHO WC measures soft tissue variations at a level less influenced by rigid bone. It is also possible that the higher (more cephalad) location of the WHO WC measurement may reflect visceral (intra-abdominal) adiposity associated with CMRFs better than the NHANES WC location. Ma et al. recently examined the difference between WC-mid (analogous to WHO) and 
WC-iliac crest (IC, analogous to NHANES) in predicting CMRFs and diabetes risk among adults in Taiwan [6]. They found that WC-mid, compared to WC-iliac crest, was more closely related to visceral fat area (by CT imaging) and to CMRFs.

Our study is the largest examining the association of NHANES and WHO WC measures with CMRFs in youth with diabetes. The SEARCH cohort is notable as the largest, U.S. population-based diabetes study among youth with racial and ethnic diversity and both diabetes types. However, this cross-sectional study does not account for changes in adiposity nor treatment effects on CMRFs over time. An additional strength is that the measurements were obtained on the same day and therefore not affected by food intake, menstrual cycle, or time of day.

These data extend our existing knowledge of the utility of measures of adiposity to a population of youth with T1D and T2D. Our results indicate that WHtR may be added as a useful indicator for assessing cardiometabolic risk. In addition, our data suggest that the WHO protocol is more strongly correlated to some CMRFs compared to the NHANES protocol in youth at certain levels of body composition.

Considering that a true association would lend itself to a uniform association between both groups, our study has a few caveats. One factor to consider is the difference in the sample size among the two diabetes types. While SEARCH has a relatively large sample of youth with T2D, the sample limits our ability to examine multiple stratifications including diabetes type. A second consideration is that there may be challenges in ascertaining accurate waist measurements particularly among larger youth, leading to greater variability and less precision in the data.

Nonetheless, our study demonstrates the association of WHtR to CMRFs in youth with diabetes and some support for a stronger association using the WHO protocol. Further research is needed to explore these relationships among youth with diabetes, including longitudinal analyses and comparisons with novel measures of cardiometabolic risk.

\section{ACKNOWLEDGEMENTS}

The SEARCH for Diabetes in Youth Study is indebted to the many youth and their families, and their health care providers, whose participation made this study possible.
All authors were involved in study design. Nora Fino and Tim Morgan analyzed the data. All authors were involved in data interpretation, generation of tables and writing the paper and had final approval of the submitted and published versions.

\section{Grant Support}

SEARCH for Diabetes in Youth is funded by the Centers for Disease Control and Prevention (PA numbers 00097, DP-05-069, and DP-10-001) and supported by the National Institute of Diabetes and Digestive and Kidney Diseases.

\section{Site Contract Numbers}

$\begin{array}{lrrr}\text { Kaiser Permanente } & \text { Southern } & \text { California } \\ \text { (U48/CCU919219, U01 DP000246, } & \text { and } \\ \text { U18DP002714), University of Colorado } & \text { Denver } \\ \text { (U48/CCU819241-3, U01 DP000247, } & \text { and }\end{array}$ U18DP000247-06A1), Children's Hospital Medical Center (Cincinnati) (U48/CCU519239, U01 DP000248, and 1U18DP002709), University of North Carolina at Chapel Hill (U48/CCU419249, U01 DP000254, and U18DP002708), University of Washington School of Medicine (U58/CCU019235-4, U01 DP000244, and U18DP002710-01), Wake Forest University School of Medicine (U48/CCU919219, U01 DP000250, and 2002010-35171).

The authors wish to acknowledge the involvement of General Clinical Research Centers (GCRC) at the South Carolina Clinical \& Translational Research (SCTR) Institute, at the Medical University of South Carolina (NIH/NCRR Grant number UL1RR029882); Seattle Children's Hospital (NIH CTSA Grant UL1 TR00423 of the University of Washington); University of Colorado Pediatric Clinical and Translational Research Center (CTRC) (Grant Number UL1 TR000154) and the Barbara Davis Center at the University of Colorado at Denver (DERC NIH P30 DK57516); and the National Center for Research Resources and the National Center for Advancing Translational Sciences, National Institutes of Health, through Grant 8 UL1 TR000077; and the Children with Medical Handicaps program managed by the Ohio Department of Health.

The findings and conclusions in this report are those of the authors and do not necessarily represent the official position of the Centers for Disease Control and Prevention and the National Institute of Diabetes and Digestive and Kidney Diseases. 
Data in this manuscript were presented in poster format at the American Diabetes Association $73^{\text {rd }}$ Scientific Session, June $21^{\text {st }}-24^{\text {th }}, 2013$, Chicago, IL.

\section{REFERENCES}

[1] Browning LM, Hsieh SD, Ashwell M. A systematic review of waist-to-height ratio as a screening tool for the prediction of cardiovascular disease and diabetes: 0.5 could be a suitable global boundary value. Nutr Res Rev 2010; 23: 247-69. http://dx.doi.org/10.1017/S0954422410000144

McCarthy HD, Ashwell M. A study of central fatness using waist-to-height ratios in UK children and adolescents over two decades supports the simple message - 'keep your waist circumference to less than half your height.' Int J Obes (London) 2006; 30; 988-92.

http://dx.doi.org/10.1038/s.ijo.0803226

[3] Kahn HS, El ghormli L, Jago R, et al. Cardiometabolic risk assessments by body mass index z-score or waist-to-height ratio in a multiethnic sample of sixth-graders. J Obes 2014; 2014: 421658. http://dx.doi.org/10.1155/2014/421658

[4] Pettitt DJ, Talton JW, Liese AD, et al. Comparison of two waist circumference measurement protocols: the SEARCH for diabetes in youth study. Pediatr Obes 2012; 7: e81-5. http://dx.doi.org/10.1111/j.2047-6310.2012.00088.x

[5] Johnson ST, Kuk JL, Mackenzie KA, Huang TT, Rosychuk RJ, Ball GD. Metabolic risk varies according to waist circumference measurement site in overweight boys and girls. J Pediatr 2010; 156: 247-52.

http://dx.doi.org/10.1016/j.jpeds.2009.08.010

[6] Ma WY, Yang CY, Shih SR, et al. Measurement of waist circumference: midabdominal or iliac crest? Diabetes Care 2013; 36: 1660-6. http://dx.doi.org/10.2337/dc12-1452

[7] SEARCH Study Group. SEARCH for Diabetes in Youth: a multicenter study of the prevalence, incidence and classification of diabetes mellitus in youth. Control Clin Trials 2004; 25: 458-71. http://dx.doi.org/10.1016/j.cct.2004.08.002
[8] Centers for Disease Control and Prevention. National Health and Nutrition Examination Survey. Anthropometry Procedures Manual (Revised December 2000): 3-30: 3-31.

[9] World Health Organization, Regional Office for Europe. Measuring obesity - Classification and description of anthropometric data: Report on a WHO Consultation on the Epidemiology of Obesity. Warsaw 1987; 2.

[10] Friedewald WT, Levy RI, Fredrickson DS. Estimation of the concentration of low-density lipoprotein cholesterol in plasma, without use of the preparative ultracentrifuge. Clin Chem 1972; 18: 499-502.

[11] Hainline A, Jr., Miller DT, Mather A. The Coronary Drug Project. Role and methods of the Central Laboratory. Control Clin Trials 1983; 4: 377-87. http://dx.doi.org/10.1016/0197-2456(83)90023-5

[12] No authors listed. Obesity and cardiovascular disease risk factors in black and white girls: the NHLBI Growth and Health Study. Am J Public Health 1992; 82: 1613-20. http://dx.doi.org/10.2105/AJPH.82.12.1613

[13] Kuczmarski RJ, Ogden CL, Guo SS, et al. 2000 CDC growth charts for the United States: methods and development, Vital and Health Statistics Series 11, no. 246, pp. 1-190, 2014. Available from: http://www.cdc.gov/growthcharts/2000 growthchart-us.pdf.]

[14] Glass GV, Stanley JC. Statistical methods in education and psychology. Prentice-Hall, Englewood Cliffs, NJ 1984.

[15] Mason C, Katzmarzyk PT. Variability in waist circumference measurements according to anatomic measurement site. Obesity (Silver Spring) 2009; 17: 1789-95. http://dx.doi.org/10.1038/oby.2009.87

[16] Patry-Parisien J, Shields M, Bryan S. Comparison of waist circumference using the World Health Organization and National Institutes of Health protocols. Health Rep 2012; 23: 53-60

[17] Wang J, Thorton JC, Bari S, et al. Comparisons of waist circumferences measured at 4 sites. Am J Clin Nutr 2003; 77: $379-84$

[18] Liu LL, Lawrence JM, Davis C, et al. Prevalence of overweight and obesity in youth with diabetes in USA: the SEARCH for Diabetes in Youth study. Pediatr Diabetes 2010; 11: 4-11.

http://dx.doi.org/10.1111/j.1399-5448.2009.00519.x 